\title{
Grain boundary assisted photocurrent collection in thin film solar cells
}

Susanna Harndt ${ }^{1}$, Christian A. Kaufmann ${ }^{1}$, Martha C. Lux-Steiner ${ }^{1}$, Reiner Klenk ${ }^{1, a}$, and Reiner Nürnberg ${ }^{2}$

${ }^{1}$ Helmholtz-Zentrum Berlin für Materialien und Energie, Hahn-Meitner-Platz 1, 14109 Berlin, Germany

${ }^{2}$ Weierstraß-Institut für Angewandte Analysis und Stochastik, Mohrenstr. 39, 10117 Berlin, Germany

Received: 25 February 2015 / Received in final form: 18 May 2015 / Accepted: 22 May 2015

Published online: 15 June 2015

(C) Harndt et al., published by EDP Sciences, 2015

\begin{abstract}
The influence of absorber grain boundaries on the photocurrent transport in chalcopyrite based thin film solar cells has been calculated using a two dimensional numerical model. Considering extreme cases, the variation in red response is more expressed than in one dimensional models. These findings may offer an explanation for the strong influence of buffer layer preparation on the spectral response of cells with small grained absorbers.
\end{abstract}

\section{Introduction}

The red response of a planar heterojunction solar cell is determined mainly by absorber properties, i.e., its absorption coefficient, doping, minority carrier diffusion length, and band gap grading. At longer wavelengths photons are absorbed at a greater distance from the hetero junction and the quantum efficiency may be reduced due to insufficient transport of minority carriers over the enlarged distance. The quantum efficiency can be calculated in analytical approximation $[1,2]$ or by numerical device modeling. Figure 1 shows a set of quantum efficiencies which have been calculated with SCAPS [3] for a typical chalcopyritebased solar cell consisting of a $\mathrm{Cu}(\mathrm{In}, \mathrm{Ga}) \mathrm{Se}_{2}$ (CIGSe) absorber, $\mathrm{CdS}$ buffer and $\mathrm{ZnO}$ window layer. Absorber doping and carrier lifetime have been varied covering the full parameter range that we expect for these cells. Absorption data (which strongly influence the result) have been taken from [4]. In contrast to this, Figure 2 shows experimental results for a set of cells where only the buffer layers were prepared differently (ILGAR with $\mathrm{InCl}_{3}$ or $\mathrm{In}(\mathrm{acac}$ ) precursor [5], chemical bath deposition of CdS, PVD of $\left.\mathrm{In}_{2} \mathrm{~S}_{3}[6]\right)$ but all the other layers were each produced in the same process. These curves are not easily understood in terms of the 1D models described above. The buffer layer by itself should have only a minor influence on carrier collection. The variation in red response in the experimental data would imply an even larger variation of absorber properties as used for the calculation in Figure 1. This suggests that buffer layer preparation somehow drastically changes the absorber properties. However, buffer prepara-

\footnotetext{
a e-mail: klenk@helmholtz-berlin.de
}

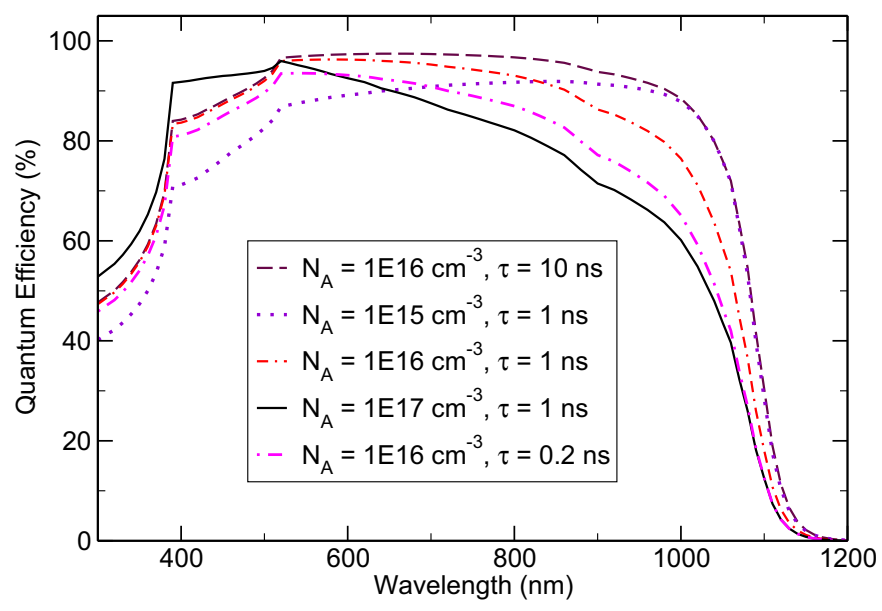

Fig. 1. Calculated quantum efficiency of CIGSe/CdS/ZnO solar cells with different shallow acceptor concentration $N_{A}$ and lifetime $\tau$ of electrons and holes in the CIGSe absorber.

tion is carried out at much lower temperature (between room temperature and $225^{\circ} \mathrm{C}$ ) than the absorber preparation which should limit such post deposition changes in absorber properties.

A possible explanation of this discrepancy involves the role of grain boundaries within the absorber for carrier collection. Diffusion of impurities along grain boundaries is much faster than within the grains and therefore could be significant even at the lower temperature used for buffer layer preparation [7]. The influence of grain boundaries must be stronger in films with smaller grains. We note that the absorbers that yielded the experiential data shown in 


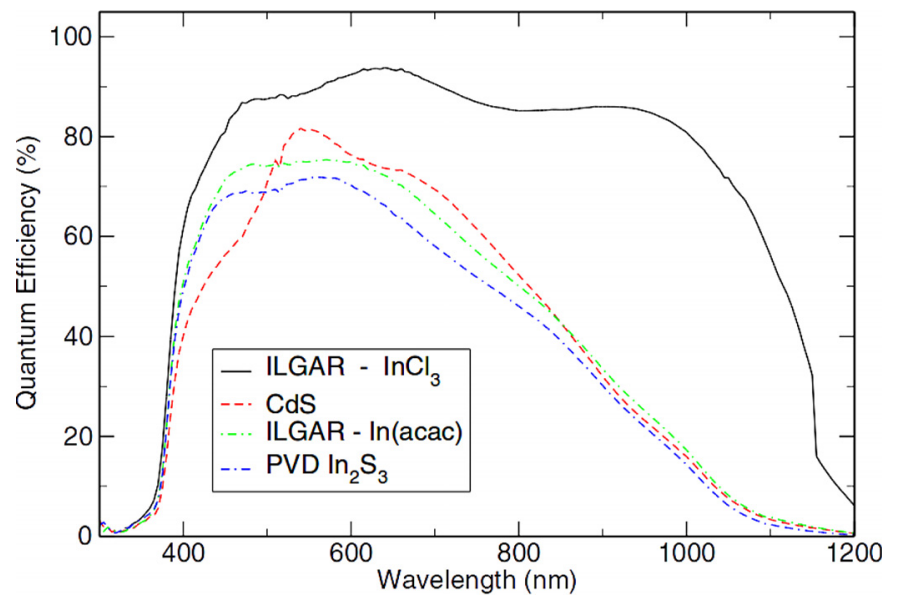

Fig. 2. Quantum efficiency of CIGSe/buffer/ZnO solar cells. All layers except the buffer were each grown in the same process. The buffer layers were deposited by ILGAR with two different precursors (ILGAR - $\mathrm{InCl}_{3}$, ILGAR - In (acac)), by chemical bath deposition (CdS) or evaporation (PVD $\left.\mathrm{In}_{2} \mathrm{~S}_{3}\right)$.

Figure 2 were grown at lower than usual substrate temperature $\left(<440{ }^{\circ} \mathrm{C}\right)$ as part of the optimization of processes for cells on polyimide foil [8] and they therefore have smaller grains. In this contribution we present $2 \mathrm{D} / 3 \mathrm{D}$ numerical modeling of grain boundary assisted photocurrent collection and discuss the results in view of grain boundaries in chalcopyrite absorbers.

\section{Calculation}

For the electronic model the grain boundary is regarded as interface (ideal plane with zero thickness) between grains. Conduction and valence band edges are continuous across the interface. Defects at the interface within the band gap cause a certain recombination velocity. In addition, defects may carry a localized charge giving rise to a symmetrical band bending towards the grain boundary. This essentially follows Seto's model [9] for grain boundaries in polycrystalline silicon. Depending on the charge distribution within the grain boundary the band edges bend "upward" (accumulation) or "downward" (depletion) from their equilibrium position within the grain towards the grain boundary. In the first case the photogenerated electrons (minority carriers in the $p$-type absorber) are driven away from the grain boundary in analogy to back surface fields used for contact passivation. In the latter case electrons are driven towards the grain boundary, potentially increasing photo current losses. However, if the depletion is further increased the charged grain boundary will eventually create a region with conductivity type inversion. The electrons are then majority carriers within the inversion zone and their recombination probability becomes very small. Furthermore, if the inversion zone connects to the $n$-type part of the heterojunction (the buffer), photo electrons can be collected by transport to and within the grain boundary. The WIASTeSCA code [10-12], which solves the semiconductor drift- diffusion equations in $2 \mathrm{D}$ or $3 \mathrm{D}$, was used to calculate the effect of grain boundary inversion on current collection. A structural unit (grain) with cylindrical symmetry was chosen so that the calculation is performed in $2 \mathrm{D}$ parameter space but provides results for an idealized 3D structure. The grain has a grain boundary around its circumference extending from the heterojunction to close to the back contact. The small gap next to the ohmic back contact was introduced to prevent shunting of the device along a path from the junction via the grain boundary to the back contact. The defects at the grain boundary can have two main consequences: deep defects act as recombination centers and charged defects cause a band bending towards the grain boundary. Our model of the defect distribution reflects these considerations by a mid-gap defect which is an effective recombination center and a donor type defect with a concentration that is sufficiently high to pin the Fermi level. Only the energy position of the latter was varied to create different band bending towards the grain boundary. All other grain boundary defect parameters were kept constant. The material properties within the grain are the same as in the SCAPS calculations shown above. The absorber thickness was set to $3 \mu \mathrm{m}$ to reduce the influence of back contact recombination. Full parameter sets are available on request from the authors.

\section{Results}

Figure 3 shows an example of a calculated potential distribution where the space charge region not only extends from the heterojunction into the absorber grain but also from its grain boundary. The direction of the electron current under illumination is given by the arrows and clearly shows the transport of electrons to the inverted grain boundary. The inversion is caused by the shallow donor type defect at the grain boundary. In Figure 4 the energy position of the donor type defect at the grain boundary has been varied. The band bending generally follows the energy of the donor. The calculated quantum efficiency at a wavelength of $1000 \mathrm{~nm}$ demonstrates the effect of grain boundaries on the red response of the solar cell. The red response is significantly enhanced for the fully inverted grain boundary (defect close to the conduction band, filled symbols), compared to the 1D case without a grain boundary (dashed lines). The quantum efficiency drops rapidly as the band bending is reduced, inversion is lost, and the hole density at the grain boundary increases. The transition occurs roughly where the band bending is equal to half of the band gap, i.e., when the defect is at mid-gap position (dotted vertical line in Fig. 4). However, close to the junction the band bending is also influenced by the buffer and window layer (Fig. 3), and a transition case exists where the grain boundary is inverted in the upper part of the grain but not in the lower part of the grain. A minimum (open symbols) is eventually reached when there still is significant band bending (depletion case of previous paragraph). As the defect level is moved deeper within the band gap, the resulting band bending does no longer follow the energy position of the defect in a 1:1 


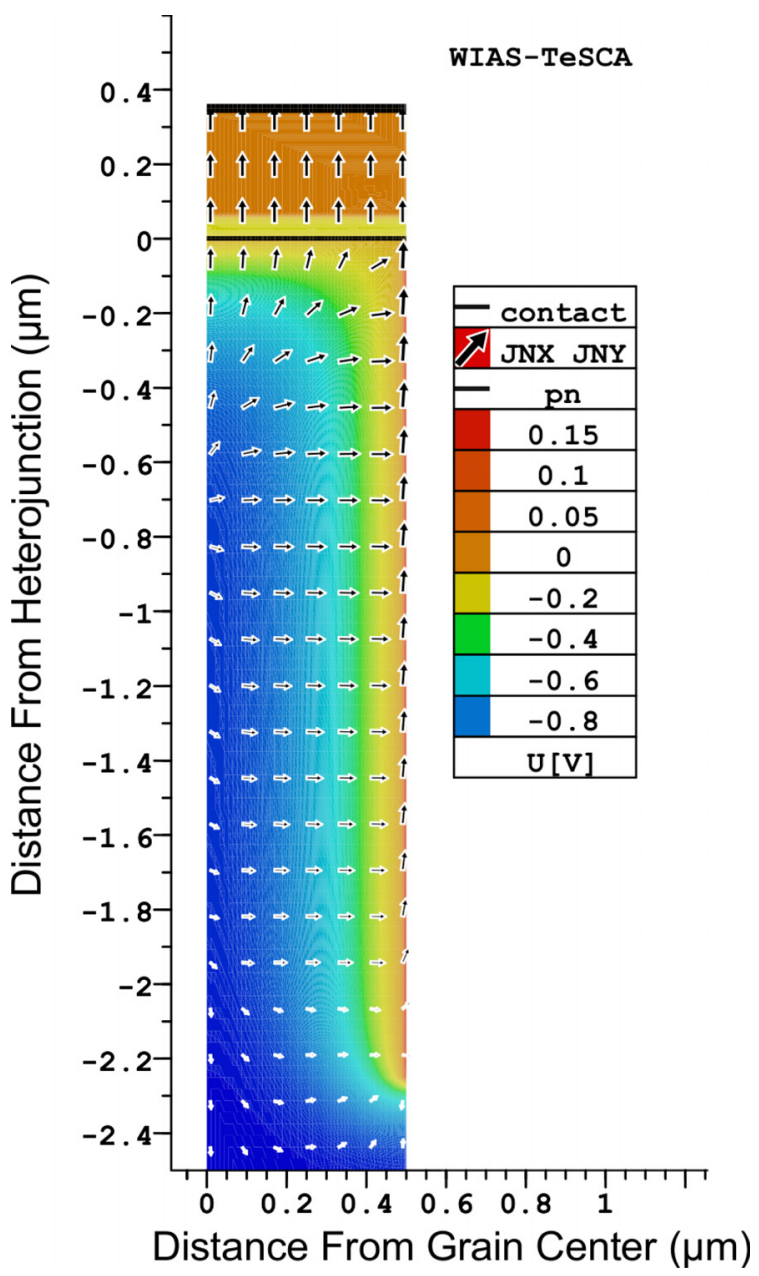

Fig. 3. Calculated potential (with respect to the equilibrium Fermi-level, color-coded) and electron current flow (arrows) in a CIGSe/CdS/ZnO structure with an inverted grain boundary in the absorber (only one half of the grain is shown). Parameters for this calculation are shallow acceptor density in the absorber $N_{A}=10^{16} \mathrm{~cm}^{-3}$, and carrier lifetimes $\tau$ of $1 \mathrm{~ns}$. The cell is illuminated with monochromatic light (wavelength $1000 \mathrm{~nm}$, power $\left.100 \mathrm{~mW} / \mathrm{cm}^{2}\right)$.

relation. This is due to incomplete pinning of the Fermi level by a single defect type. Consequently, the accumulation case is not reached in these calculations. Qualitatively, the results are very similar for two different levels of absorber doping, however, the effects are more significant for the higher doping level. The influence of grain boundaries on red response for varied absorber properties can be seen in Figure 5. Looking at, e.g., the emphasized black curves, the red response can be significantly higher (filled symbols) as well as lower (open symbols) as compared to the 1D case without grain boundaries (dashed lines). Furthermore, both gain and loss depend on the absorber bulk properties (doping and carrier lifetime). As expected, both maximum gain (inverted grain boundaries) as well as loss in quantum efficiency are reduced with increasing grain size. However, even at a grain radius of $2 \mu \mathrm{m}$ there still is a significant influence of the grain boundaries, at least

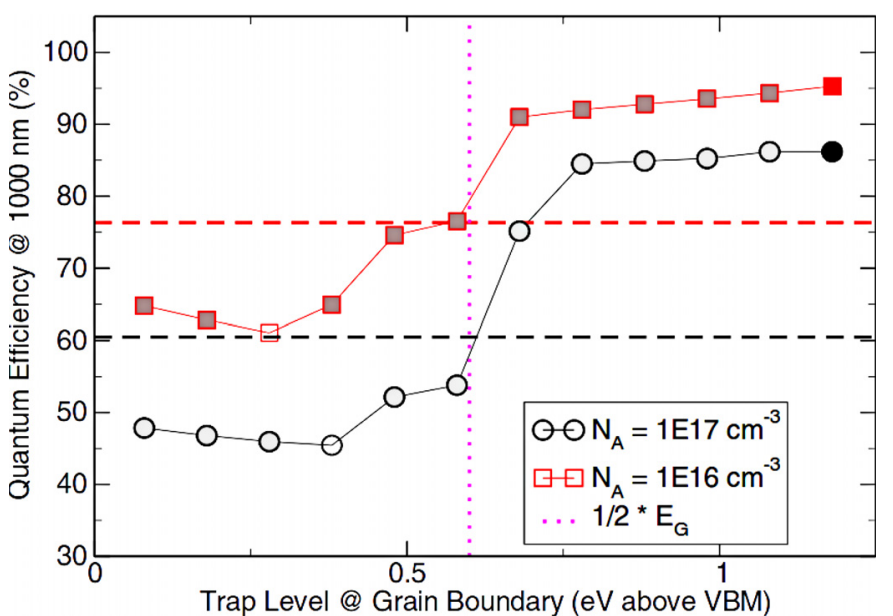

Fig. 4. Calculated quantum efficiency of polycrystalline CIGSe/CdS/ZnO solar cells at a wavelength of the incident light of $1000 \mathrm{~nm}$. The energy position of the donor type defect at the grain boundary (trap level) has been varied to modify the band bending towards the grain boundary. The dashed horizontal lines indicate the quantum efficiency without grain boundary (1D case). The dotted vertical line marks the midgap position. The absorber grain radius is $1 \mu \mathrm{m}$ and the carrier lifetimes are 1 ns.

for the extreme cases regarded here. Figure 6 shows quantum efficiencies over the full wavelength range (in analogy to Figs. 1 and 2) for the three grain boundary configurations (fully inverted grain boundaries, without grain boundary, maximum recombination). Results calculated with the WIAS-TeSCA code for the 1D case without grain boundaries are identical to those calculated with SCAPS. The discrepancy at lower wavelengths is only due to neglecting the optical absorption in the window and buffer layer in the WIAS-TeSCA model.

\section{Discussion}

The calculations confirm that the defect distribution (modeled here by just one mid-gap recombination center and one donor with varied energy) at grain boundaries is an additional and quite important parameter in the design of efficient thin film solar cells even when compared to other parameters such as grain size, absorber doping, and minority carrier lifetime. Measurements of device quality chalcopyrite films [13] have found depletion zones at grain boundaries and it was suggested that these may lead to improved carrier collection [14]. Current transport along grain boundaries has also been measured by conductive scanning probe microscopy $[15,16]$. With respect to the current collection, our calculations show that there can be a negative as well as a positive influence depending on the band bending towards the grain boundary. The reported band bending would be too small to achieve the beneficial effect on current collection predicted by our model, however, it has been argued that the measurements underestimate the band bending at grain boundaries due 


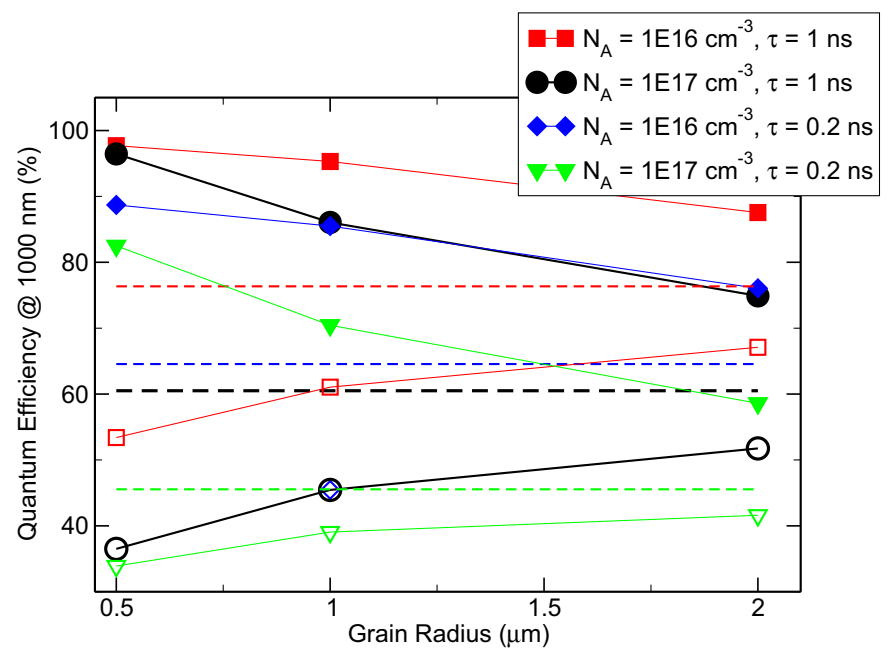

Fig. 5. Calculated quantum efficiency of polycrystalline CIGSe/CdS/ZnO solar cells at a wavelength of the incident light of $1000 \mathrm{~nm}$ as a function of absorber grain radius. Filled symbols indicate inverted grain boundaries whereas empty symbols refer to the minimum quantum efficiency that is reached for a specific trap level (see previous figure). The dashed lines indicate the quantum efficiency without grain boundary (1D case).

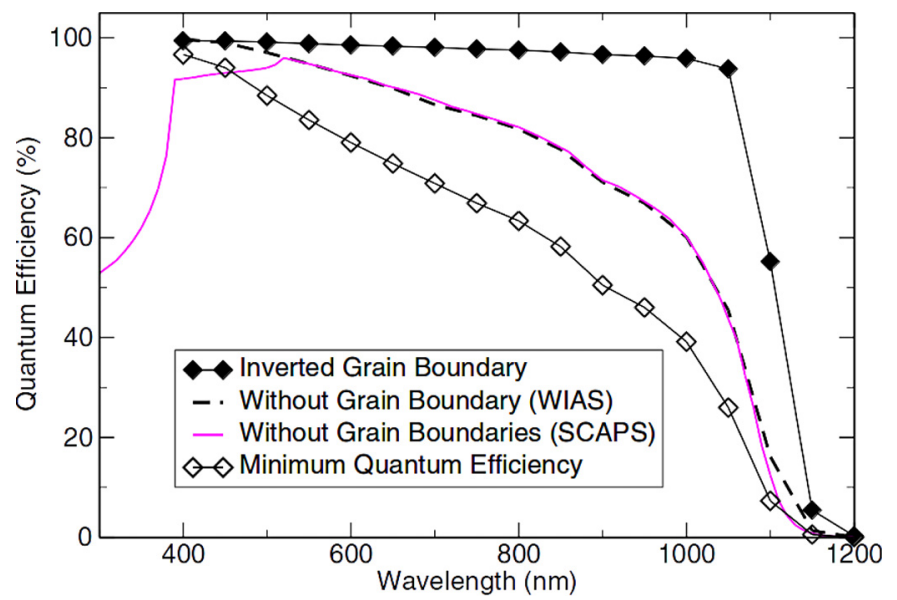

Fig. 6. Calculated spectral quantum efficiency of CIGSe/CdS/ZnO solar cells. Acceptor density and lifetime in the absorber are $N_{A}=10^{17}$ and $\tau=1 \mathrm{~ns}$, respectively. The absorber grain radius is $0.5 \mu \mathrm{m}$.

to surface band bending [13] and the averaging effect of the scanning probe [17].

Taretto et al. [18] limited their numerical calculations to the case of depleted grain boundaries and have thus shown only the negative influence of grain boundary recombination. Our finding of both, negative and positive influence, is in principle agreement with calculations described in reference [19]. However, the authors only reported the effect on the total photo current density under white light illumination and did not show the quantum efficiency at different wavelengths. An increase in white light induced photo current for inverted grain boundaries was also found in reference [20]. A different electronic model of the grain boundary has been proposed where $\mathrm{Cu}$ depletion leads to a discontinuity of the valence band (hole barrier) $[21,22]$. The previous calculations in references $[18,19]$ also show some results for this alternative model. It is clear that such a hole barrier would decrease the recombination of electrons at the grain boundaries even under the conditions which lead to poor carrier collection in our calculations. In summary, the influence of material properties on the spectral response would be closer to the $1 \mathrm{D}$ model (without grain boundaries) [20].

In terms of overall device performance, the optimum band bending towards the grain boundaries depends on other parameters such as carrier lifetime and doping. If the minority carrier diffusion length within the grains is sufficient for carrier collection, an accumulation layer towards the grain boundary is preferable to the inverted grain boundary. This is due to the decreased open circuit voltage of devices with inverted grain boundaries. The significance of this loss depends on the dominating recombination mechanism giving rise to the bucking current. Also, depending on how strongly the Fermi-level is pinned at the grain boundary, the inversion may vanish at higher forward bias, resulting in strongly voltage-dependent carrier collection (poor fill factor). In material with grains smaller than considered here the grain interior may become depleted and the band bending may be insufficient to establish inverted grain boundaries. For low quality material, the trade-off between improved current collection and reduced open circuit voltage may be optimized in the numerical model but difficult to achieve in the experiment. It was concluded in reference [20] that in most cases the performance of a CIGSe based cell cannot be improved by grain boundaries. The geometry of a device with grain boundary assisted carrier collection is in some respects similar to that of the ETA (Extremely Thin Absorber) solar cell. Analytical modeling [23] of this cell demonstrates both the potential as well as the complexity of adapting the geometry to achieve reasonable efficiencies with limited material quality.

If we consider again the large variation in red response in cells with different buffer layers (Fig. 1), it is indeed conceivable from the calculations that absorber grain boundaries could play a significant role. Together with the fast diffusion of impurities along grain boundaries, this suggests that process steps carried out after absorber preparation and at significantly lower temperature, such as the deposition of buffer and window layers, can still influence absorber and hence device properties. Here, a particularly good red response has been observed when the buffer layer was deposited with ILGAR using a chlorine containing precursor solution. It has been suggested that $\mathrm{Cl}$ may cause donor type defects $\left(\mathrm{Cl}_{\mathrm{Se}}\right)$ in the bulk of $\mathrm{CuInSe}_{2}[24,25]$. We speculate that during buffer deposition $\mathrm{Cl}$ is diffusing along the grain boundaries, leads to inverted grain boundaries and superior carrier collection. The expected corresponding loss in open circuit voltage is also systematically observed in the experiment when using buffers prepared from the $\mathrm{InCl}_{3}$ solution. Nevertheless, 
it should be understood that the experimental observation has mainly been the motivation to revisit the problem of grain boundary assisted current collection. Additional work would be required to establish the claim that ILGAR deposition of a buffer layer with the $\mathrm{Cl}$ containing precursor leads to inverted grain boundaries in the absorber. The additional work could include measurements of electron beam induced current to map the electron collection, assessment of the $\mathrm{Cl}$ concentration at the grain boundary, and direct measurement of the band bending by Kelvin probe microscopy.

\section{Conclusions}

Numerical calculations show that grain boundaries in the absorber can have a large influence on photocurrent collection. Hence, the defect distribution (recombination centers, charged defects) at grain boundaries is an additional and quite important parameter in the design of efficient thin film solar cells, even when compared to other parameters such as grain size, absorber doping, and minority carrier lifetime. Shallow donor type defects at the grain boundary can cause an inversion zone in which electrons are transported leading to very high quantum efficiency even in films with high doping and short carrier lifetimes. When the defect is located deeper in the band gap, the inversion and carrier transport are lost causing excessive recombination losses. These findings are a tentative explanation for the expressed differences in quantum efficiency which were found for cells with differently prepared buffer layers.

Financial support by the German Federal Ministry of Economy and Technology (BMWi), represented by the German Aerospace Center (DLR) under Contract 50RN1101 is gratefully acknowledged. The authors thank Adrien Bercegol for discussions.

\section{References}

1. R. Klenk, H.-W. Schock, in Proceedings of the 12th European Photovoltaic Solar Energy Conference, Amsterdam, 1994, pp. 1588-1591

2. M. Troviano, K. Taretto, Sol. Energy Mater. Sol. Cells 95, 821 (2011)

3. M. Burgelman, P. Nollet, S. Degrave, Thin Solid Films 361, 527 (2000)

4. M. Gloeckler, A. Fahrenbruch, J. Sites, in Proceedings of the 3rd World Conference on Photovoltaic Energy Conversion, Osaka, 2003, pp. 491-494
5. R. Sáez-Araoz, J. Krammer, S. Harndt, T. Koehler, M. Krueger, P. Pistor, A. Jasenek, F. Hergert, M.Ch. LuxSteiner, Ch.-H. Fischer, Prog. Photovolt.: Res. Appl. 20, $855(2012)$

6. P. Pistor, R. Caballero, D. Hariskos, V. Izquierdo-Roca, R. Wächter, S. Schorr, R. Klenk, Sol. Energy Mater. Sol. Cells 93, 148 (2009)

7. M. Rusu, M. Bär, S. Lehmann, S. Sadewasser, L. Weinhardt, C.A. Kaufmann, E. Strub, J. Röhrich, W. Bohne, I. Lauermann, Ch. Jung, C. Heske, M.Ch. LuxSteiner, Appl. Phys. Lett. 95, 173502 (2009)

8. R. Caballero, C.A. Kaufmann, T. Eisenbarth, A. Eicke, Th. Unold, R. Klenk, H.-W. Schock, Mater. Res. Soc. Symp. Proc. 1165, M02-10 (2009)

9. J.Y.W. Seto, J. Appl. Phys. 48, 5247 (1975)

10. WIAS TeSCA - Modeling and Simulation of Semiconductor Devices, http://www.wias-berlin.de/software/tesca, last accessed on January 6th, 2015

11. H. Gajewski, Mitt. Ges. Angew. Math. Mech. 16, 35 (1993)

12. H. Gajewski, H.-Chr. Kaiser, H. Langmach, R. Nürnberg, R.H. Richter, Mathematical Modeling and Numerical Simulation of Semiconductor Detectors, in Mathematics Key Technology for the Future, edited by W. Jäger, H.-J. Krebs (Springer, New York, 2003), pp. 355-364

13. C.-S. Jiang, R. Noufi, J.A. AbuShama, K. Ramanathan, H.R. Moutinho, J. Pankow, M.M. Al-Jassim, Appl. Phys. Lett. 84, 3477 (2004)

14. C.-S. Jiang, R. Noufi, K. Ramanathan, J.A. AbuShama, H.R. Mouthino, M.M. Al-Jassim, Appl. Phys. Lett. 85, 2625 (2004)

15. D. Azulay, O. Millo, I. Baalberg, H.-W. Schock, I. VisolyFisher, D. Cahen, Sol. Energy Mater. Sol. Cells 91, 85 (2007)

16. S. Sadewasser, D. Abou-Ras, D. Azulay, R. Baier, I. Balberg, D. Cahen, S. Cohen, K. Gartsman, K. Ganesan, J. Kavalakkatt, W. Li, O. Millo, Th. Rissom, Y. Rosenwaks, H.-W. Schock, A. Schwarzman, T. Unold, Thin Solid Films 519, 7341 (2011)

17. R. Baier, C. Leendertz, D. Abou-Ras, M.C. Lux-Steiner, Sol. Energy Mater. Sol. Cells 130, 124 (2014)

18. K. Taretto, U. Rau, J.H. Werner, Thin Solid Films 480481, 8 (2005)

19. M. Gloeckler, J.R. Sites, W.K. Metzger, J. Appl. Phys. 98, $113704(2005)$

20. K, Taretto, U. Rau, Mater. Res. Soc. Symp. Proc. 1012, 309 (2007)

21. M.J. Hetzer, Y.M. Strzhemechny, M. Gao, M.A. Contreras, A. Zunger, L.J. Brillson, Appl. Phys. Lett. 86, 162105 (2005)

22. C. Persson, A. Zunger, Phys. Rev. Lett. 91, 266401 (2003)

23. K. Taretto, U. Rau, Prog. Photovolt.: Res. Appl. 12, 573 (2004)

24. T. Tanaka, T. Yamaguchi, T. Ohshima, H. Itoh, A. Wakahara, A. Yoshida, Sol. Energy Mater. Sol. Cells 75, $109(2003)$

25. Y.-J. Zhao, C. Persson, S. Lany, A. Zunger, Appl. Phys. Lett. 85, 5860 (2004) 